\title{
Minimum Vessel Lumen Diameter
}

National Cancer Institute

\section{Source}

National Cancer Institute. Minimum Vessel Lumen Diameter. NCI Thesaurus. Code

C116144.

The minimum diameter of the inside of a blood vessel (lumen) derived quantitatively in a single view. 